\title{
Get Screened: A Randomized Trial of the Incremental Benefits of Reminders, Recall, and Outreach on Cancer Screening
}

\author{
Robert J. Fortuna, $M D, M P H^{7}$, Amna ldris, $\mathrm{MPH}^{2}$, Paul Winters, $\mathrm{MS}^{2}$, \\ Sharon G. Humiston, MD, MPH $H^{3}$, Steven Scofield, MD', Samantha Hendren, MD, MPH', \\ Patricia Ford, MS ${ }^{2}$, Shirley X. L. Li, B SciH $H^{2}$, and Kevin Fiscella, MD, MPH $H^{2,5}$
}

\begin{abstract}
${ }^{1}$ Center for Primary Care and Department of Internal Medicine, Culver Medical Group, University of Rochester School of Medicine and Dentistry, Rochester, NY, USA; ${ }^{2}$ Department of Family Medicine and Community \& Preventive Medicine, University of Rochester School of Medicine and Dentistry, Rochester, NY, USA; ${ }^{3}$ Department of Pediatrics, Children's Mercy Hospitals and Clinics, Kansas City, MO, USA; ${ }^{4}$ Department of Surgery, University of Michigan, Ann Arbor, MI, USA; ${ }^{5}$ Family Medicine Research Programs, University of Rochester, Rochester, NY, USA.
\end{abstract}

BACKGROUND: Rates of breast cancer (BC) and colorectal cancer (CRC) screening are particularly low among poor and minority patients. Multifaceted interventions have been shown to improve cancer-screening rates, yet the relative impact of the specific components of these interventions has not been assessed. Identifying the specific components necessary to improve cancer-screening rates is critical to tailor interventions in resource limited environments.

OBJECTIVE: To assess the relative impact of various components of the reminder, recall, and outreach (RRO) model on $\mathrm{BC}$ and $\mathrm{CRC}$ screening rates within a safety net practice.

DESIGN: Pragmatic randomized trial.

PARTICIPANTS: Men and women aged 50-74 years past due for CRC screen and women aged 40-74 years past due for BC screening.

INTERVENTIONS: We randomized 1,008 patients to one of four groups: (1) reminder letter; (2) letter and automated telephone message (Letter + Autodial); (3) letter, automated telephone message, and point of service prompt (Letter + Autodial + Prompt); or (4) letter and personal telephone call (Letter + Personal Call).

MAIN MEASURES: Documentation of mammography or colorectal cancer screening at 52 weeks following randomization.

KEY RESULTS: Compared to a reminder letter alone, Letter + Personal Call was more effective at improving screening rates for $\mathrm{BC}(17.8 \%$ vs. $27.5 \%$; AOR 2.2, $95 \%$ CI 1.2-4.0) and CRC screening (12.2\% vs. $21.5 \%$; AOR 2.0, 95 \% CI 1.1-3.9). Compared to letter alone, a Letter + Autodial + Prompt was also more effective at improving rates of $\mathrm{BC}$ screening (17.8\% vs. $28.2 \%$; AOR 2.1, $95 \%$ CI 1.1-3.7) and CRC screening (12.2\% vs. $19.6 \%$; AOR 1.9, $95 \%$ CI 1.0-3.7). Letter + Autodial was not more effective than a letter alone at improving screening rates.

Electronic supplementary material The online version of this article (doi:10.1007/s11606-013-2586-y) contains supplementary material, which is available to authorized users.

Received March 1, 2013

Revised July 9, 2013

Accepted August 7, 2013

Published online September 4, 2013
CONCLUSIONS: The addition of a personal telephone call or a patient-specific provider prompt were both more effective at improving mammogram and CRC screening rates compared to a reminder letter alone. The use of automated telephone calls, however, did not provide any incremental benefit to a reminder letter alone.

KEY WORDS: cancer screening; colorectal cancer; breast cancer; outreach; inreach.

J Gen Intern Med 29(1):90-7

DOI: $10.1007 / \mathrm{s} 11606-013-2586-\mathrm{y}$

() Society of General Internal Medicine 2013

\section{BACKGROUND}

Screening for colorectal cancer (CRC) and breast cancer (BC) are key strategies towards reducing mortality and morbidity from these diseases. Although effective screening strategies are commonly available, many patients delay or forego screening. Rates of cancer screening, notably mammography and CRC screening are particularly low among poor and some minority patients. ${ }^{1}$ Although overall rates of screening have improved over the past 15 years, screening rates remain well below national objectives set by Healthy People 2020. ${ }^{1}$

Multiple interventions have been studied to improve cancer-screening rates, but the optimal methods have yet to be defined. A recent review from the Community Preventive Services Task Force found strong evidence to support client reminders, such as letters and phone calls, to improve both breast cancer and CRC screening. ${ }^{2}$ Prior studies have demonstrated that a letter alone increased screening rates for BC by approximately $4.5-9 \%$ and for CRC by $0.2-10.9 \%{ }^{2,3}$ More recently, several studies have examined the benefit of multimodal interventions, including the RRO model to remind patients and providers when screening is due, recall unscreened patients, and implement culturally appropriate outreach to patients in need of screening. ${ }^{4-6}$ Although multifaceted interventions have been shown to improve 
cancer-screening rates, ${ }^{5-7}$ the relative incremental impact of the specific components of these interventions have not been fully assessed. Identifying the specific components necessary to improve cancer-screening rates is critical to tailor effective interventions in resource-limited environments.

We previously conducted a trial of a multimodal intervention to increase cancer screening in a similar safety-net practice and found a significant increase in screening rates, ${ }^{7}$ raising the question which component(s) of the intervention were most important. This project was designed to assess the relative impact of various components of the reminder, recall, and outreach (RRO) model on $\mathrm{BC}$ and $\mathrm{CRC}$ screening rates within a safety-net practice. Our primary aim was to assess the incremental benefit, beyond a single reminder letter, of automated telephone calls, point of service prompts, and personal phone calls to increase cancer-screening rates within a safety net practice. We used a pragmatic randomized trial to test the hypothesis that the addition of these interventions to mailed letters would provide incremental increases in cancer screening.

\section{METHODS}

\section{Practice Setting}

We examined the RRO model in an urban internal medicine practice in Rochester, New York, a city of 211,000 inhabitants (37.6\% non-Hispanic white, $41.7 \%$ black). ${ }^{8}$ The study practice cared for a largely low-income and disproportionately minority patient population, and was situated in an urban federal-designated underserved setting. The study site was an academic practice located in the community with eight attending physicians and 28 resident physicians.

\section{Patient Identification and Eligibility}

Potentially eligible participants were identified using practice-billing data and a query of the electronic health system based on age, gender and last visit to the practice. We used a single download from the office electronic medical record (EMR) system to establish a practice registry. Research staff conducted individual patient EMR reviews to confirm that all patients were still active patients and to identify last BC and CRC screening date and cancer risk-status. Research staff manually reviewed 1,567 charts to determine eligibility (Fig. 1).

Inclusion Criteria. Participant eligibility criteria for enrollment in the study included: (1) being a registered patient at the study clinic; (2) being an active patient at the practice (having at least one visit to the practice in the last 2 years); (3) being female age 40-74 for BC screening; (4) being age 50 to 74 for CRC screening; (5) being past due for $\mathrm{BC}$ or CRC screening. We defined past due for mammography as $>18$ months from last mammogram. This 18 -month period was based on recommendations from American Cancer Society plus a 6-month grace period. ${ }^{9,10}$ We defined past due for CRC as $>10$ years from past colonoscopy (unless recommended earlier by the colonoscopist) or, in the absence of colonoscopy results, $>12$ months from past fecal occult blood test or fecal immunochemical test (FOBT/FIT) or $>5$ years from last double contrast barium enema or flexible sigmoidoscopy. Women ages 50 to 74 could be enrolled based on either (or both) BC or CRC criteria. We used these standards, based on American Cancer Society, ${ }^{9,10}$ unless the patient's primary care provider had indicated an alternate screening interval in the chart.

Exclusion Criteria. Patients were excluded from the study if they were at higher risk for cancer. Conditions signaling higher risk of cancer included prior cancer, premalignant conditions (e.g., ulcerative colitis, familial polyposis), inadequately evaluated breast masses, positive FOBT/FIT result at last testing, or a first-degree relative with a previous diagnosis of $\mathrm{BC}$ or CRC. Higher risk patients who were not up-to-date with recommended screening were notified separately of their need for screening.

\section{Randomization of Patients}

After eligibility was determined, an offsite study statistician, who was blinded to the identity of the patient, assigned participants equally into one of the four intervention groups, using a random number algorithm, stratified by the type of screening(s) required (BC, CRC or both). Allocation was concealed. Figure 1 demonstrates randomization and patient flow through the clinical protocol. Patients who were eligible for mammography and CRC interventions were randomized once, and received interventions targeting both mammography and CRC. Healthcare personnel and study staff were unaware of group assignment.

\section{Intervention Groups}

A detailed timeline for the four intervention groups is available in the Online Appendix Figure 1.

Group \#1: All groups were sent a single letter from the practice using the patient's most current available home address from the medical record. The letter, with a personalized salutation, indicated to the patient that $\mathrm{s} / \mathrm{he}$ was overdue for screening and included information regarding the importance of screening and how to schedule screening. The letter provided the name and telephone number of the outreach worker available to provide assistance with scheduling mammography or arranging colonoscopy referrals. The letter also indicated that free 


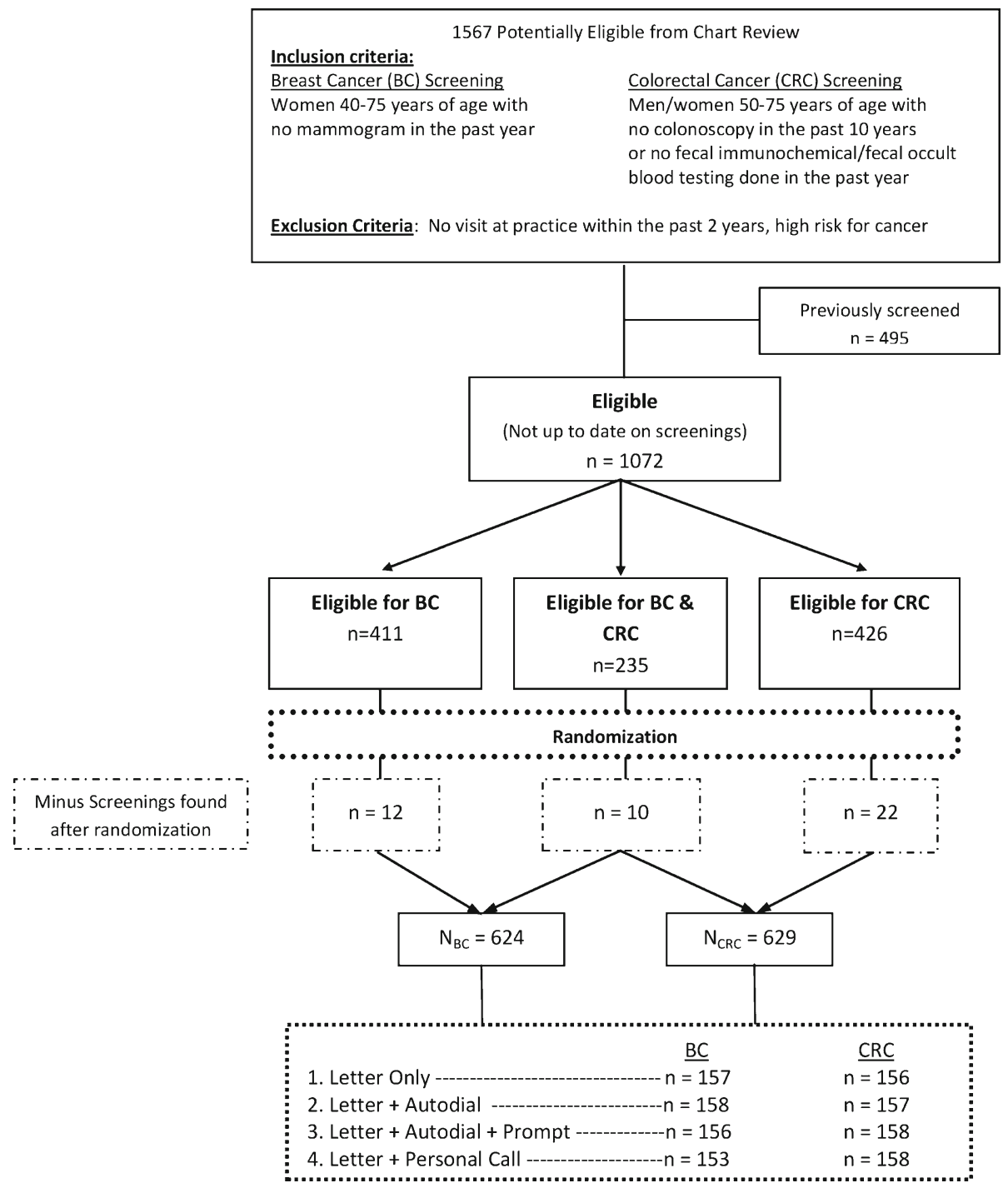

Figure 1. Patient randomization flow chart.

screening for uninsured/underinsured patients was available through a state sponsored program. Letters were available in English and Spanish. A sample letter appears in the appendix (Online Appendix Figure 2). Subjects in Group $\# 1$ received no other intervention.

Group \#2: The Letter + Autodial group received the letter (described above), plus a series of up to five automated telephone calls. We contracted with Televox ${ }^{\circledR}$ for these calls. We used the patient's most current available telephone number from the medical record. Telephone calls were attempted for up to 2 weeks at varying times throughout the day/evening until a person or an answering machine responded. The automated message contained similar information to the letter, with instructions to call the outreach worker or the practice to arrange for screening or with questions. These calls were delivered to patients on weeks 2 and 8 following randomization. Until there was documented screening, chart reviews were performed on weeks 12 and 26. Automated telephone messages were repeated on weeks 14,28 , and 38 for patients remaining unscreened at these time periods. The automated telephone call messages are available in the Online Appendix.

Group \#3: The Letter + Autodial + Prompt group received the same intervention as Group \#2 plus paper prompts delivered at the time of a patient-initiated visit. We used paper prompts (Online Appendix 3) because this enabled us to deliver similar prompts to patients and clinicians simultaneously, and because of doubts regarding effects of electronic prompts on clinician screening. ${ }^{11}$ Research staff reviewed scheduling modules weekly to check for planned acute and preventive visits by patients in Group \#3. Prompts were delivered to the treating clinician at the point of care to remind the patient and provider about overdue screening. Prompts were provided at both acute and preventive visits. The back of each CRC prompt sheet briefly summarized advantages and limitations for CRC 
screening modalities as a way of facilitating clinicianpatient discussion. The prompt addressed both colonoscopy and FIT kits.

Group \#4: The Letter + Personal Call group received the letter described for Group \#1 plus a personal telephone call from a trained outreach worker. These telephone calls were attempted up to three times, at varying times of the day and varying days of the week, with a 1-week period between attempts. When/if the patient was reached, the outreach worker explained that she was calling on behalf of the practice to remind the patient that $\mathrm{s} / \mathrm{he}$ was overdue for cancer screening. She used motivational interviewing principles to encourage screening and offered assistance with scheduling an appointment, as well as relevant telephone numbers and logistical assistance, including referral(s) for free mammography and FIT for the uninsured. Patients that did not want to undergo a colonoscopy were offered a mailed FIT kit as an alternative method of CRC screening. If a patient refused to have any screening tests done for $\mathrm{BC}$ or $\mathrm{CRC}$, it was indicated in the patient registry and interventions were stopped.

\section{Targeting Non-Responders and Blinded Review of Records}

In order to target the iterative intervention solely to nonresponders and minimize unnecessary contact with patients who already had obtained cancer screening, we conducted two interim chart reviews. Specifically, at weeks 12 and 26 of the intervention period, staff blinded to group assignment reviewed the EMR of all randomized subjects (regardless of group assignment) to assess if cancer screening had been completed. If cancer screening was completed or the patient had transferred his/her care to another practice, the intervention and future EMR reviews were stopped. If, during an interim review, a screening test was discovered that was dated prior to the date of randomization, it was indicated in the database, and interventions and all future EMR reviews were stopped. All subjects were analyzed in the originally assigned study group.

\section{Data Collection}

Independent Variables. We obtained each patient's age, sex, race/ethnicity, insurance status, and household zip code from the EMR. We assigned each patient a household income based on the median household income for his/her zip code, as abstracted from the 2000 US Census.

Primary Outcome. The primary outcome was EMR documentation of mammography or colorectal cancer screening at 52 weeks based on the presence of a test report with results following randomization (approximately 3 months after the end of interventions). This time period, which extended 14 weeks beyond the last intervention in any group, was designed to allow documentation of any screenings initiated late in the intervention period to show up in the EMR. The final EMR review was conducted at week 52 by research staff blinded to intervention group. Patients were considered 'screened' if documentation was found for $\mathrm{BC}$ (mammography report) or CRC (FOBT, FIT, colonoscopy, flexible sigmoidoscopy, or double contrast barium enema reports) according to the original standards.

\section{Statistical Analysis}

We performed all statistical analyses using SAS version 9.3 (SAS Institute Inc., Cary, NC, USA). All subjects were analyzed in the originally assigned study group, based on intention-to-treat. We compared post intervention screening rates between the four randomization groups using chci square test statistics. We used logistic regression models to evaluate the incremental effect of the RRO model while controlling for age, sex, race/ethnicity, insurance status, and household income.

This study was approved by the University of Rochester Research Subjects Review Board (RSRB00031463).

\section{RESULTS}

A total of the 1,567 patients initially met the base eligibility criteria of age and active status in the practice. Of these, 1,072 (68 \%) were past due for BC and/or CRC screening and met the eligibility criteria for randomization. Figure 1 shows the patient flow through the clinical protocol. A total of 624 patients met final BC eligibility criteria and 629 met final CRC eligibility criteria. The baseline demographic characteristics of the randomized patients are shown in Tables 1 and 2. There were no significant differences in participants at baseline between the four intervention groups for either BC or CRC participants.

Tables 3 and 4 describe the crude and adjusted odds ratios for the intervention groups for mammography and CRC screening participants. Compared to a reminder letter alone, Letter + Personal Call was more effective at improving screening rates for both $\mathrm{BC}(17.8 \%$ vs. $27.5 \%$; AOR 2.2, $95 \%$ CI 1.2-4.0) and CRC screening (12.2\% vs. $21.5 \%$; AOR 2.0, CI 1.1-3.9). Compared to a reminder letter alone, Letter + Autodial + Prompt also was more effective at improving rates of $\mathrm{BC}(17.8 \%$ vs. $28.2 \%$; AOR 2.1, CI 1.1-3.7) and CRC screening (12.2 vs. $19.6 \%$; AOR 1.9, CI 
Table 1. Demographic Characteristics of Mammography Screening Participants by Intervention Group

\begin{tabular}{|c|c|c|c|c|}
\hline \multirow[t]{2}{*}{ Characteristic } & \multicolumn{4}{|c|}{ Intervention Group } \\
\hline & $\begin{array}{l}\text { Letter \% } \\
(n=157)\end{array}$ & $\begin{array}{l}\text { Letter + Automated } \\
\text { Call } \%(n=158)\end{array}$ & $\begin{array}{l}\text { Letter + Automated } \\
\text { Call + Visit Prompt \% }(n=156)\end{array}$ & $\begin{array}{l}\text { Letter + Personal } \\
\text { Call } \%(n=153)\end{array}$ \\
\hline \multicolumn{5}{|l|}{ Age (years) } \\
\hline $40-49$ & $47.1 \%(74)$ & $50.6 \%(80)$ & $46.8 \%(73)$ & $53.09 \%(81)$ \\
\hline $50-59$ & $29.9 \%(47)$ & $27.2 \%(43)$ & $29.5 \%(46)$ & $32.0 \%(49)$ \\
\hline $60+$ & $22.9 \%(36)$ & $22.2 \%(35)$ & $23.7 \%(37)$ & $15.0 \%(23)$ \\
\hline Race/ethnicity & $n=129$ & $n=132$ & $n=128$ & $n=127$ \\
\hline Non-Hispanic Black & $43.4 \%(56)$ & $40.2 \%(53)$ & $36.7 \%(47)$ & $36.2 \%(46)$ \\
\hline Other (including Hispanic) & $10.9 \%(14)$ & $12.1 \%(16)$ & $21.1 \%(27)$ & $16.5 \%(21)$ \\
\hline Non-Hispanic White & $45.7 \%(59)$ & $47.7 \%(63)$ & $42.2 \%(54)$ & $47.3 \%(60)$ \\
\hline Insurance & $n=156$ & $n=158$ & $n=155$ & $n=152$ \\
\hline Private & $39.1 \%(61)$ & $36.0 \%(57)$ & $40.7 \%(63)$ & $37.5 \%(57)$ \\
\hline Medicaid & $30.1 \%(47)$ & $34.2 \%(54)$ & $31.0 \%(48)$ & $33.6 \%(51)$ \\
\hline Medicare & $23.7 \%(37)$ & $22.8 \%(36)$ & $21.9 \%(34)$ & $21.7 \%(33)$ \\
\hline None & $7.1 \%(11)$ & $7.0 \%(11)$ & $6.4 \%(10)$ & $7.2 \%(11)$ \\
\hline Household income* & $n=156$ & $n=156$ & $n=156$ & $n=153$ \\
\hline$\$ 40,000+$ & $24.5 \%(37)$ & $28.6 \%(47)$ & $29.6 \%(47)$ & $27.6 \%(44)$ \\
\hline$\$ 30,000-\$ 39,000$ & $38.0 \%(62)$ & $43.4 \%(67)$ & $41.3 \%(63)$ & $44.3 \%(68)$ \\
\hline$<\$ 30,000$ & $36.5 \%(57)$ & $26.9 \%(42)$ & $29.5 \%(46)$ & $26.8 \%(41)$ \\
\hline
\end{tabular}

Column percentages reported

*Household income based on the median household income for the subject's zip code, as abstracted from the 2000 US Census

1.0-3.7). Compared to a reminder letter alone, Letter + Autodial was not more effective at increasing mammogram or CRC screening.

Personal telephone calls were attempted up to three times. The patient navigator was successful in reaching $65.9 \%$ of patients randomized to receive a personal call, not including messages left. Within Group \#4, those who were reached with a call more likely to receive screening compared to those who were not reached with a call (30.9 \% vs. $20.2 \% ; P=0.05)$.
Colonoscopy screening was the most common CRC screening modality used. FOBT or FIT screening accounted for $5.3 \%$ of CRC screening in the letter group, $16.7 \%$ in the Letter + Autodial group, $10.7 \%$ in the Letter + Autodial + Prompt group, and 38.2\% in the Letter + Personal Call group.

We did not find any significant treatment group by covariate interactions in an exploratory analysis. A few subjects had $\mathrm{BC}$ and $\mathrm{CRC}$ screenings that were found after randomization (Fig. 1). The distribution of these patients

Table 2. Demographic Characteristics of Colorectal Cancer Screening Participants by Intervention Group

\begin{tabular}{|c|c|c|c|c|}
\hline \multirow[t]{2}{*}{ Characteristic } & \multicolumn{4}{|c|}{ Intervention Group } \\
\hline & $\begin{array}{l}\text { Letter \% } \\
(n=156)\end{array}$ & $\begin{array}{l}\text { Letter + Automated } \\
\text { Call } \%(n=157)\end{array}$ & $\begin{array}{l}\text { Letter + Automated } \\
\text { Call + Visit Prompt \% }(n=158)\end{array}$ & $\begin{array}{l}\text { Letter + Personal } \\
\text { Call } \%(n=158)\end{array}$ \\
\hline \multicolumn{5}{|l|}{ Sex } \\
\hline Female & $51.9 \%(81)$ & $56.7 \%(89)$ & $55.1 \%(87)$ & $53.8 \%(85)$ \\
\hline Male & $48.1 \%(75)$ & $43.3 \%(68)$ & $44.9 \%(71)$ & $46.2 \%(73)$ \\
\hline Age (years) & $n=156$ & $n=157$ & $n=158$ & $n=158$ \\
\hline $50-59$ & $63.5 \%(99)$ & $59.2 \%(93)$ & $63.3 \%(100)$ & $64.6 \%(102)$ \\
\hline $60+$ & $36.5 \%(57)$ & $40.8 \%(64)$ & $36.7 \%(58)$ & $35.4 \%(56)$ \\
\hline Race/ethnicity & $n=136$ & $n=135$ & $n=133$ & $n=127$ \\
\hline Non-Hispanic Black & $33.1 \%(45)$ & $35.6 \%(48)$ & $37.6 \%(50)$ & $34.7 \%(44)$ \\
\hline Other (including Hispanic) & $14.0 \%(19)$ & $12.6 \%(17)$ & $12.0 \%(16)$ & $17.3 \%(22)$ \\
\hline Non-Hispanic White & $52.9 \%(72)$ & $51.8 \%(70)$ & $50.4 \%(67)$ & $48.0 \%(61)$ \\
\hline Insurance & $n=156$ & $n=157$ & $n=158$ & $n=158$ \\
\hline Private & $34.6 \%(54)$ & $33.1 \%(52)$ & $41.1 \%(65)$ & $39.9 \%(63)$ \\
\hline Medicaid & $26.9 \%(42)$ & $26.1 \%(41)$ & $24.7 \%(39)$ & $20.3 \%(32)$ \\
\hline Medicare & $32.1 \%(50)$ & $33.1 \%(52)$ & $26.0 \%(41)$ & $33.5 \%(53)$ \\
\hline None & $6.4 \%(10)$ & $7.6 \%(12)$ & $8.2 \%(13)$ & $6.3 \%(10)$ \\
\hline Household income & $n=155$ & $n=157$ & $n=157$ & $n=157$ \\
\hline$\$ 40,000+$ & $29.7 \%(46)$ & $29.3 \%(46)$ & $29.9 \%(47)$ & $26.7 \%(42)$ \\
\hline$\$ 30,000-\$ 39,000$ & $36.1 \%(56)$ & $40.1 \%(63)$ & $44.0 \%(69)$ & $39.5 \%(62)$ \\
\hline$<\$ 30,000$ & $34.2 \%(53)$ & $30.6 \%(48)$ & $26.1 \%(41)$ & $33.8 \%(53)$ \\
\hline
\end{tabular}

Column percentages reported 
Table 3. Crude and Adjusted Odds Ratios for Mammography Participants

\begin{tabular}{|c|c|c|c|}
\hline Characteristic & Screening rates & Crude Odds Ratio (CI) & Adjusted Odds Ratio (CI) \\
\hline \multicolumn{4}{|l|}{ Intervention Group } \\
\hline 1: Letter & $17.8 \%$ & 1.0 & 1.0 \\
\hline 2: Letter + Autodial & $22.8 \%$ & $1.4(0.8-2.4)$ & $1.3(0.7-2.4)$ \\
\hline 3: Letter + Autodial + Prompt & $28.2 \%$ & $1.8(1.1-3.1)$ & $2.1(1.1-3.7)$ \\
\hline 4: Letter + Personal Call & $27.5 \%$ & $1.7(1.0-3.0)$ & $2.2(1.2-4.0)$ \\
\hline \multicolumn{4}{|l|}{ Age (years) } \\
\hline $40-49$ & $23.7 \%$ & $0.9(0.6-1.5)$ & $1.2(0.7-1.9)$ \\
\hline $50-59$ & $23.8 \%$ & $0.9(0.6-1.6)$ & $1.4(0.8-2.6)$ \\
\hline $60+$ & $25.2 \%$ & 1.00 & 1.00 \\
\hline \multicolumn{4}{|l|}{ Race/ethnicity } \\
\hline Non-Hispanic Black & $29.2 \%$ & $1.3(0.9-2.0)$ & $1.3(0.8-2.1)$ \\
\hline Other (including Hispanic) & $26.9 \%$ & $1.2(0.7-2.1)$ & $1.0(0.6-1.9)$ \\
\hline Non-Hispanic White & $23.7 \%$ & 1.00 & 1.00 \\
\hline \multicolumn{4}{|l|}{ Insurance } \\
\hline Medicaid & $26.5 \%$ & $1.2(0.8-1.8)$ & $1.2(0.7-1.9)$ \\
\hline Medicare & $25.0 \%$ & $1.1(0.7-1.8)$ & $1.1(0.6-1.9)$ \\
\hline None & $14.0 \%$ & $0.5(0.2-1.3)$ & $0.5(0.2-1.3)$ \\
\hline Private & $23.5 \%$ & 1.00 & 1.00 \\
\hline \multicolumn{4}{|l|}{ Household income* } \\
\hline$\$ 40,000+$ & $19.4 \%$ & $0.7(0.4-1.1)$ & $0.7(0.4-1.3)$ \\
\hline$\$ 30,000-\$ 39,000$ & $25.4 \%$ & $1.0(0.6-1.5)$ & $1.0(0.6-1.6)$ \\
\hline$<\$ 30,000$ & $25.8 \%$ & 1.00 & 1.00 \\
\hline
\end{tabular}

*Household income based on the median household income for the subject's zip code, as abstracted from the 2000 US Census

did not differ among study groups for BC screening $(p<0.48)$ or CRC screening $(p<0.97)$.

\section{DISCUSSION}

Screening for colorectal and breast cancers are key strategies towards reducing mortality and morbidity from these diseases, yet rates of screening remain below national targets. Numerous strategies exist to improve screening rates for colorectal and breast cancer, $2,3,5,6,11-20$ but the relative incremental benefit of these interventions have not been compared. ${ }^{5}$ Using a pragmatic randomized trial, we examined the incremental benefit of several strategies to augment the effect of a patient reminder letter to improve cancer-screening rates. We found that personal telephone calls to patients (outreach) and personalized point-of-care reminders to providers (inreach) both provided additional benefit to the baseline reminder letter. An automated telephone call, however,

Table 4. Crude and Adjusted Odds Ratios for Colorectal Cancer Screening Participants

\begin{tabular}{|c|c|c|c|}
\hline Effect & Screening rates & Crude Odds Ratio (CI) & Adjusted Odds Ratio (CI) \\
\hline \multicolumn{4}{|l|}{ Intervention group } \\
\hline 1: Letter & $12.2 \%$ & 1.00 & 1.00 \\
\hline 2: Letter + Autodial & $15.3 \%$ & $1.3(0.7-2.5)$ & $1.2(0.6-2.4)$ \\
\hline 3: Letter + Autodial + Prompt & $19.6 \%$ & $1.8(1.0-3.3)$ & $1.9(1.0-3.7)$ \\
\hline 4: Letter + Personal Call & $21.5 \%$ & $2.0(1.1-3.7)$ & $2.0(1.1-3.9)$ \\
\hline \multicolumn{4}{|l|}{ Sex } \\
\hline Female & $19.6 \%$ & $1.5(1.1-2.2)$ & $1.6(1.0-2.6)$ \\
\hline Male & $14.3 \%$ & 1.00 & 1.00 \\
\hline \multicolumn{4}{|l|}{ Age (years) } \\
\hline $50-59$ & $17.3 \%$ & 1.00 & 1.00 \\
\hline $60+$ & $17.0 \%$ & $1.0(0.6-1.5)$ & $1.2(0.7-1.9)$ \\
\hline \multicolumn{4}{|l|}{ Race/ethnicity } \\
\hline Non-Hispanic Black & $17.1 \%$ & $1.0(0.6-1.6)$ & $1.0(0.6-1.7)$ \\
\hline Other (including Hispanic) & $25.7 \%$ & $1.6(0.9-3.0)$ & $1.9(1.0-3.7)$ \\
\hline Non-Hispanic White & $17.4 \%$ & 1.00 & 1.00 \\
\hline \multicolumn{4}{|l|}{ Insurance } \\
\hline Medicaid & $15.6 \%$ & $0.8(0.4-1.3)$ & $0.9(0.5-1.6)$ \\
\hline Medicare & $15.8 \%$ & $0.8(0.5-1.3)$ & $0.7(0.4-1.2)$ \\
\hline None & $15.6 \%$ & $0.8(0.3-1.8)$ & $0.5(0.2-1.5)$ \\
\hline Private & $19.7 \%$ & 1.00 & 1.00 \\
\hline \multicolumn{4}{|l|}{ Household income } \\
\hline$\$ 40,000+$ & $17.1 \%$ & $1.1(0.7-2.0)$ & $1.2(0.6-2.4)$ \\
\hline$\$ 30,000-\$ 39,000$ & $18.4 \%$ & $1.2(0.8-2.1)$ & $1.4(0.8-2.4)$ \\
\hline$<\$ 30,000$ & $15.4 \%$ & 1.00 & 1.00 \\
\hline
\end{tabular}

*Household income based on the median household income for the subject's zip code, as abstracted from the 2000 US Census 
did not provide any additional benefit over a reminder letter alone.

As the national health care policy looks towards the patientcentered medical home to improve care-coordination and screening, ${ }^{21}$ effective strategies are needed to efficiently augment rates of cancer screening without duplicating efforts. Our study provides evidence that personalized outreach (personal reminder telephone call) and directed in-reach (patient-specific provider prompts) were both effective strategies to augment a reminder letter to improve CRC and mammography rates. The personal nature of each of these interventions, compared to an automated approach, likely contributed to their success. Our results support those of previous studies. ${ }^{11,22-24}$ Green et al. recently found that an automated mailing led to twice as many persons being screened for colorectal cancers. ${ }^{23}$ Adding navigated interventions, such as guided support from a registered nurse, led to further improvements in CRC screening rates. ${ }^{23}$ Additional studies have also demonstrated the benefit of point of care prompts $^{11,12}$ and personal telephone calls to improve cancer screening rates among women. ${ }^{22}$

In contrast to personalized outreach and in-reach, we found that automated telephone calls did not provide additional benefit beyond a reminder letter. Automated telephone messages are widely used for many purposes, and have been shown to improve attendance at healthcare appointments and adherence to medications. ${ }^{25}$ However, it is possible that patients require a more personalized approach when contemplating decisions surrounding cancer screening. Both personal telephone calls and provider prompts offer that opportunity for discussion surrounding cancer screening that may be necessary to further boost screening rates beyond a reminder letter alone. Similarly, a recent systematic review found insufficient evidence to support 'mass media' outreach to improve breast and colorectal cancer screening. ${ }^{2}$

Improving the coordination of health care and boosting evidence-based cancer screening rates are key strategies of evolving health policy. The patient-centered medical home offers the potential infrastructure to provide care-coordination and implement components of the RRO model - to remind patients and providers when screening is due, recall unscreened patients, and perform outreach to patients in need of screening. Both personalized outreach (personal reminder telephone call) and directed in-reach (patient-specific provider prompts) were effective in our study and could be incorporated into a medical home with a care-manager. Our outreach worker functioned within the scope of most embedded care-managers in medical homes. ${ }^{26}$ Similar to embedded care-managers ${ }^{26}$ and patientnavigators, ${ }^{23,27}$ our outreach worker used motivational interviewing principles to encourage screening and offered logistical assistance with scheduling an appointment. Moving forward, the expansion of EMRs into patient-centered medical homes offers the potential to further implement components of the RRO model into routine practice.
This study had several limitations. First, this study occurred in a single large internal medicine safety-net practice located in an urban undeserved area. Overall, the baseline screening rates were relatively low, but similar to other safety net practices in the surrounding area. Given the relatively low baseline rates, it is possible that other settings with higher baseline rates may not experience the same improvement as seen in this study. Conversely, settings in which the patient telephone numbers recorded in the EMR are more up-to-date may experience even greater rate increases. Second, it is possible that some patients may have undergone screening without having supporting documentation in the EMR. Every aspect of the EMR was examined for screening reports, including scanned media from outside institutions. Further, there is no reason to believe that any possible missed screenings would be different between the intervention groups. Third, we did not assess the costs of each intervention. Multiple cost analyses have been published in the past, ${ }^{28-33}$ and analysis of the cost-perincremental screening will be an important consideration in future studies. It is also worth noting that this study followed guidelines from the American Cancer Society that were in place in 2010. These guidelines were applied equally to all study groups, but differ slightly from the recent U.S. Preventive Services Task Force (USPSTF) guidelines.

\section{CONCLUSION}

As the national health care policy looks towards the patientcentered medical home to improve care coordination and screening, effective and efficient strategies are needed to improve cancer-screening rates. We found that personalized outreach (personal reminder telephone call) and directed inreach (patient-specific point-of-care provider prompts) were both more effective at improving breast and colorectal cancer screening rates compared to a reminder letter alone. The use of automated telephone calls did not provide any incremental benefit to a reminder letter alone.

\section{Acknowledgments:}

Contributors: All authors listed have contributed sufficiently to the project to be included as authors, and all those who are qualified to be authors are listed in the author byline.

Grant Support: This project was supported by the American Cancer Society - RSGT-08-077-01-CPHPS.

Conflict of Interest: The authors declare that they do not have a conflict of interest.

Corresponding Author: Kevin Fiscella, MD, MPH; Family Medicine Research Programs, University of Rochester 1381 South Avenue, Rochester, NY 14620, USA (e-mail: Kevin_Fiscella@URMC.Rochester.edu). 


\section{REFERENCES}

1. Cancer screening-United States, 2010. MMWR Morb Mortal Wkly Rep. 2012;61(3):41-5.

2. Sabatino SA, Lawrence B, Elder R, et al. Effectiveness of interventions to increase screening for breast, cervical, and colorectal cancers: nine updated systematic reviews for the guide to community preventive services. Am J Prev Med. 2012;43(1):97-118. doi:10.1016/j.amepre.2012.1004.1009.

3. Brouwers MC, De Vito C, Bahirathan L, et al. What implementation interventions increase cancer screening rates? A systematic review. Implement Sci. 2011;6:111. doi:10.1186/1748-5908-1186-1111.

4. Rodewald LE, Szilagyi PG, Humiston SG, Barth R, Kraus R, Raubertas RF. A randomized study of tracking with outreach and provider prompting to improve immunization coverage and primary care. Pediatrics. 1999;103(1):31-8.

5. Fiscella K, Humiston S, Hendren S, et al. A multimodal intervention to promote mammography and colorectal cancer screening in a safety-net practice. J Natl Med Assoc. 2011;103(8):762-8.

6. Fiscella K, Yosha A, Hendren SK, et al. Get screened: a pragmatic randomized controlled trial to increase mammography and colorectal cancer screening in a large, safety net practice. BMC Health Serv Res. 2010;10:280. doi:10.1186/1472-6963-1110-1280.

7. Hendren S, Winters P, Humiston S, et al. Randomized controlled trial of a multimodal intervention to improve cancer screening rates in a safety-net primary care practice. JGIM. 2013. doi:10.1007/s11606-0132506-1.

8. U.S. Census Bureau: State and County QuickFacts. Data derived from Population Estimates, American Community Survey, Census of Population and Housing, County Business Patterns, Economic Census, Survey of Business Owners, Building Permits, Consolidated Federal Funds Report, Census of Governments 2012; http://quickfacts.census.gov/ qfd/states/36/3663000.html. Accessed 7/27/2013.

9. Gaseem A, Denberg TD, Hopkins RH Jr, et al. Screening for colorectal cancer: a guidance statement from the American College of Physicians. Ann Intern Med. 2012;156(5):378-86.

10. Smith RA, Cokkinides V, Brooks D, Saslow D, Brawley OW. Cancer screening in the United States, 2010: a review of current American Cancer Society guidelines and issues in cancer screening. CA Cancer $J$ Clin. 2010;60(2):99-119.

11. Sequist TD, Zaslavsky AM, Marshall R, Fletcher RH, Ayanian JZ. Patient and physician reminders to promote colorectal cancer screening: a randomized controlled trial. Arch Intern Med. 2009;169(4):364-71. doi:10.1001/archinternmed.2008.1564.

12. Baron RC, Melillo S, Rimer BK, et al. Intervention to increase recommendation and delivery of screening for breast, cervical, and colorectal cancers by healthcare providers a systematic review of provider reminders. Am J Prev Med. 2010;38(1):110-7. doi:10.1016/ j.amepre.2009.1009.1031

13. Khankari K, Eder M, Osborn CY, et al. Improving colorectal cancer screening among the medically underserved: a pilot study within a federally qualified health center. J Gen Intern Med. 2007;22(10):1410-4.

14. Ling BS, Schoen RE, Trauth JM, et al. Physicians encouraging colorectal screening: a randomized controlled trial of enhanced office and patient management on compliance with colorectal cancer screening. Arch Intern Med. 2009;169(1):47-55.

15. Lasser KE, Murillo J, Lisboa S, et al. Colorectal cancer screening among ethnically diverse, low-income patients: a randomized controlled trial. Arch Intern Med. 2011;171(10):906-12.
16. Segnan N, Senore C, Andreoni B, et al. Randomized trial of different screening strategies for colorectal cancer: patient response and detection rates. J Natl Cancer Inst. 2005;97(5):347-57.

17. Sequist TD, Zaslavsky AM, Colditz GA, Ayanian JZ. Electronic patient messages to promote colorectal cancer screening: a randomized controlled trial. Arch Intern Med. 2011;171(7):636-41. doi:10.1001/ archinternmed.2010.1467. Epub 2010 Dec 1013.

18. Swan J, Breen N, Coates RJ, Rimer BK, Lee NC. Progress in cancer screening practices in the United States: results from the 2000 National Health Interview Survey. Cancer. 2003;97(6):1528-40.

19. Feldstein AC, Perrin N, Rosales AG, et al. Effect of a multimodal reminder program on repeat mammogram screening. Am J Prev Med. 2009;37(2):94-101.

20. Phillips CE, Rothstein JD, Beaver $\mathbf{K}$, Sherman BJ, Freund KM, Battaglia TA. Patient navigation to increase mammography screening among inner city women. J Gen Intern Med. 2011;26(2):123-9.

21. Agency for Healthcare Research and Quality. Patient Centered Medical Home Resource Center. http://pcmh.ahrq.gov. Accessed 7/ 27/2013.

22. Dietrich AJ, Tobin JN, Cassells A, et al. Telephone care management to improve cancer screening among low-income women: a randomized, controlled trial. Ann Intern Med. 2006;144(8):563-71.

23. Green BB, Wang CY, Anderson ML, et al. An automated intervention with stepped increases in support to increase uptake of colorectal cancer screening: a randomized trial. Ann Intern Med. 2013;158(5 Pt 1):301-11. doi:10.7326/0003-4819-7158-7325-201303050201300002.

24. Kempe KL, Shetterly SM, France EK, Levin TR. Automated phone and mail population outreach to promote colorectal cancer screening. Am J Manag Care. 2012;18(7):370-8.

25. Car J, Gurol-Urganci I, de Jongh T, Vodopivec-Jamsek V, Atun R. Mobile phone messaging reminders for attendance at healthcare appointments. Cochrane Database Syst Rev. 2012;7, CD007458.

26. Hines P, Mercury M. Designing the role of the embedded care manager. Prof Case Manag. 2013;18(4):182-7.

27. Freeman HP. The history, principles, and future of patient navigation: commentary. Semin Oncol Nurs. 2013;29(2):72-5.

28. Ishikawa $\mathbf{Y}$, Hirai $\mathbf{K}$, Saito $\mathbf{H}$, et al. Cost-effectiveness of a tailored intervention designed to increase breast cancer screening among a nonadherent population: a randomized controlled trial. BMC Publ Health. 2012;12:760. doi:10.1186/1471-2458-1112-1760.

29. Lee JK, Groessl EJ, Ganiats TG, Ho SB. Cost-effectiveness of a mailed educational reminder to increase colorectal cancer screening. BMC Gastroenterol. 2011;11:93. doi:10.1186/1471-1230X-1111-1193.

30. Misra S, Lairson DR, Chan w, et al. Cost effectiveness of interventions to promote screening for colorectal cancer: a randomized trial. J Prev Med Public Health. 2011;44(3):101-10. doi:10.3961/jpmph.2011. 3944.3963.3101.

31. Saywell RM Jr, Champion VL, Skinner CS, Menon U, Daggy J. A cost-effectiveness comparison of three tailored interventions to increase mammography screening. J Womens Health (Larchmt). 2004; 13(8):909-18.

32. Sequist TD, Franz C, Ayanian JZ. Cost-effectiveness of patient mailings to promote colorectal cancer screening. Med Care. 2010;48(6):553-7. doi:10.1097/MLR. 1090b1013e3181dbd1098eb.

33. Smith DH, Feldstein AC, Perrin N, et al. Automated telephone calls to enhance colorectal cancer screening: economic analysis. Am J Manag Care. 2012;18(11):691-9. 